OSU-TA-24/94

(December 29, 1994)

\title{
THE X-RAY CLUSTER BARYON CRISIS
}

\author{
Gary Steigman \\ Departments of Physics and Astronomy, The Ohio State University \\ 174 West 18th Avenue, Columbus, OH 43210 \\ and \\ James E. Felten \\ Code 685, NASA Goddard Space Flight Center \\ Greenbelt, MD 20771
}

\begin{abstract}
Nucleosynthesis in the standard hot big bang cosmology offers a successful account of the production of the light nuclides during the early evolution of the Universe. Consistency among the predicted and observed abundances of $\mathrm{D},{ }^{3} \mathrm{He},{ }^{4} \mathrm{He}$ and ${ }^{7} \mathrm{Li}$ leads to restrictive lower and upper bounds to the present density of nucleons. In particular, the upper bound ensures that nucleons cannot account for more than a small fraction $\left(<0.06 h_{50}^{-2}\right)$ of the mass in a critical density (Einstein-de Sitter) Universe. In contrast, x-ray observations of rich clusters of galaxies suggest strongly that baryons (in hot gas) contribute a significant fraction of the total cluster mass $\left(\geq 0.2 h_{50}^{-3 / 2}\right)$. If, indeed, clusters do provide a "fair" sample of the mass in the Universe, this "crisis" forces us to consider other ways of mitigating it, including the politically incorrect possibility that $\Omega<1$. The options, including magnetic or turbulent pressure, clumping, and non-zero space curvature and/or cosmological constant, are discussed.
\end{abstract}

To appear in Proceedings of the St. Petersburg Gamow Seminar (Sept. 12-14, 1994), ed. A. M. Bykov \& R. A. Chevalier; Sp. Sci. Rev., in press (Dordrecht: Kluwer). 


\section{INTRODUCTION}

The standard, hot big bang cosmology provides a successful model of an expanding Universe filled with radiation. As Gamow and his collaborators Alpher and Herman realized, the Universe described by this model would have passed through an early, hot, dense epoch when nuclear reactions transformed neutrons and protons into the light nuclides deuterium, helium-3, helium-4 and lithium-7 (e.g., Boesgaard \& Steigman 1985).

The primordial abundances predicted by Big Bang Nucleosynthesis (BBN) in the standard model depend on only one adjustable parameter - the nucleon density at BBN. For relatively high nucleon density models (as measured by the nucleon-tophoton ratio $\eta=n_{N} / n_{\gamma} ; \eta_{10} \equiv 10^{10} \eta$ ), nucleosynthesis begins early, when neutrons are relatively abundant. In this case, $\mathrm{D}$ and ${ }^{3} \mathrm{He}$ are quickly burned to ${ }^{4} \mathrm{He}$, and it is easier to bridge the gap at mass-5 and produce relatively large yields of mass- 7 . Thus for "high" $\eta$ the primordial abundances of D and ${ }^{3} \mathrm{He}$ are "small" while those of ${ }^{4} \mathrm{He}$ and ${ }^{7} \mathrm{Li}$ are "large". In contrast, for relatively low nucleon density models, the start of BBN is somewhat delayed, permitting some neutrons to decay and resulting in a somewhat less efficient burning of deuterium and helium-3 (as well as lithium-7). So, for "low" $\eta$ the big bang yields of D and ${ }^{3} \mathrm{He}$ (as well as ${ }^{7} \mathrm{Li}$ ) are "large" while that of "He is "small". With four predicted abundances (relative to hydrogen) and only one adjustable parameter, the standard, hot big bang cosmology is a testable model. As a function of $\eta$ the predicted BBN yields range over some 10 orders of magnitude. So, too, do the "observed" primordial abundances as inferred from a wide diversity of astronomical observations [e.g., Walker et al. ("WSSOK") 1991; for a recent overview, see Steigman 1994a]. For quite some time now it has been known that theory and data are roughly consistent (at the $\sim 2$-sigma level) provided that the nucleon abundance lies in a very narrow range: $2.8 \lesssim \eta_{10} \lesssim 4.0$ [WSSOK; or: $3.1 \lesssim \eta_{10} \lesssim 3.9$ (Steigman 1994a) .

It is, of course, not sufficient to find that a value (or narrow range of values) of $\eta$ exists such that BBN predicts correctly the primordial abundances of the light nuclides (although any cosmological model must pass this test). It is necessary, too, to see if the nucleon abundance $\eta$ inferred from processes in the youth of the Universe is consistent with that determined from observations in its maturity. Astronomical data exist on the dynamics of systems from galaxies to clusters of galaxies (and beyond), from which estimates of the universal mass density may be derived. The BBN inferred nucleon mass density must be compared with those estimates to further test the standard, hot big bang cosmology.

For convenience (as well as convention) we will use for our comparisons the density parameter $\Omega$, the ratio of the present mass density to the critical mass density $\rho_{\text {crit }}$.

$$
\rho_{\text {crit }}=\frac{3 H_{0}^{2}}{8 \pi G} \approx 2.6 h_{50}^{2} \mathrm{keVcm}^{-3}
$$


In (1), $G$ is Newton's constant and we have written the Hubble constant as: $H_{0}=$ $50 h_{50} \mathrm{~km} \mathrm{~s}^{-1} \mathrm{Mpc}^{-1}$. The nucleon-to-photon ratio $\eta$ (e.g., from BBN) and the present photon (Cosmic Background Radiation $\equiv \mathrm{CBR}$ ) temperature determine the present universal nucleon density. For $T_{C B R} \approx 2.73 \mathrm{~K}$,

$$
\Omega_{B B N} h_{50}^{2} \approx 0.015 \eta_{10}
$$

[For $T_{C B R}=2.726 \pm 0.010 \quad$ (Mather et al. 1994), $\Omega_{B B N} h_{50}^{2} / \eta_{10}=0.0146_{-0.0001}^{+0.0002}$. Thus, for $2.8 \lesssim \eta_{10} \lesssim 4.0$ (see Fig. 1 ),

$$
0.04 \lesssim \Omega_{B B N} h_{50}^{2} \lesssim 0.06
$$

We show this allowed range of $\Omega_{B B N}$ as a function of $H_{0}$ in Figure 1 . For comparison, we show also an estimate of $\Omega_{L U M}$, the contribution to $\Omega$ by "luminous" baryonic matter; i.e., baryonic matter within optically visible galaxies. We obtained this value by assuming, within the luminous parts of galaxies, a mean ratio of baryonic mass to blue luminosity $\left\langle M / L_{B}\right\rangle=7.5 h_{50}$, and dividing by the critical ratio needed to obtain $\Omega=1,\left(M / L_{B}\right)_{\text {crit }}=750 h_{50}$, inferred from surveys of the large-scale luminosity density (Efstathiou, Ellis, \& Peterson 1988). This estimate $\Omega_{L U M}=10^{-2}$ is generous. Some would argue that it is an upper bound.

Comparing $\Omega_{B B N}$ and $\Omega_{L U M}$, we see that as required for consistency, BBN has provided evidence for at least as many nucleons as observed today $\left(\Omega_{B B N} \geq \Omega_{L U M}\right.$ for $\left.H_{0} \leq 100 \mathrm{~km} \mathrm{~s}^{-1} \mathrm{Mpc}^{-1}\right)$. Indeed, for $H_{0}<100$, the gap in Fig. 1 between $\Omega_{B B N}$ and $\Omega_{L U M}$ provides evidence that a significant fraction of all nucleons in the Universe may be "dark" (baryonic dark matter) - at least in the sense of not being within the optically visible parts of galaxies.

This argument has been made before. We should offer two cautionary remarks: (1) Our generous chosen ratio $\left\langle M / L_{B}\right\rangle=7.5 h_{50}$ [cf. the value $3.2 h_{50}$ used by White et al. (1993)] comes from dynamical determinations (as shown by the $H_{0}$-dependence) and therefore includes all gravitating mass within the images studied, not just baryonic mass. If some fraction of this mass were non- baryonic, our estimate for baryonic mass $\Omega_{L U M}=10^{-2}$ would have to be decreased. (2) There are few dynamical measurements in the outer parts of galaxies. If large amounts of baryonic matter were present there, the number $10^{-2}$ might have to be increased. We shall discuss this further elsewhere (Felten \& Steigman 1994).

We also show in Figure 1 a lower bound $\left(\Omega_{D Y N}>0.1\right)$ to the total mass density inferred from gravitational dynamics on the scales of clusters and beyond. If $H_{0}$ is not very small (e.g., for $H_{0} \geq 40$ ), we see that $\Omega_{B B N}<\Omega_{D Y N}$, implying that the universal mass density contains, and may be dominated by, non-baryonic dark matter ("The Ultimate Copernican Principle"!). 


\section{CONSISTENCY OR CRISIS ?}

The dramatic quantitative success of BBN provides strong support for the standard, hot big bang cosmology. At the same time, however, it requires that we proceed to a higher level of accuracy in testing the consistency of this model. As we move on to more stringent tests, recent observational data have revealed three potential crises looming on the horizon (Steigman 1994a).

(a) Is the Predicted ${ }^{4} \mathrm{He}$ Yield Too High?

The lower bound to $\eta$ and, therefore, to the BBN predicted yield of ${ }^{4} \mathrm{He}$, is derived (solely!) from a upper bound to primordial D and/or ${ }^{3} \mathrm{He}$ derived from solar system data (Yang et al. 1984; Dearborn, Schramm, \& Steigman 1986; WSSOK; Steigman \& Tosi 1992, 1994). For $\eta_{10} \geq 2.8$ (WSSOK) and the standard case of three light neutrino species $\left(N_{\nu}=3\right)$ and a neutron lifetime $\geq 885 \mathrm{sec}$, the predicted BBN ${ }^{4} \mathrm{He}$ mass fraction is "large": $Y_{B B N} \geq 0.241$. In contrast, the primordial abundance inferred from observations of low metallicity, extragalactic $\mathrm{H}$ II regions (Pagel et al. 1992, Skillman et al. 1993) is "small". The recent analysis of this large data sample by Olive \& Steigman (1994) concludes that $Y_{P}=0.232 \pm 0.003$; the uncertainty is the 1- $\sigma$ statistical error in the mean. In the absence of any systematic uncertainties, the 2- $\sigma_{\text {stat }}$ upper bound is inconsistent with the theoretical lower bound. However, this potential crisis may be avoided with allowance for a modest $(\sim 2 \%)$ systematic uncertainty in the observationally inferred $Y_{P}$. Sources of such uncertainties could be small corrections for unseen neutral helium, for collisional excitation, for corrections due to radiation trapping in the presence of dust, or for errors in the atomic emissivities. Although Olive \& Steigman (1994) and Pagel (1993) have estimated that $\sigma_{\text {syst. }} \sim 0.005$, Copi, Schramm, \& Turner (1994) have suggested much larger uncertainties $\sim 0.016$. The resolution of this potential crisis will require careful analyses of the data from the most metal-poor extragalactic $\mathrm{H}$ II regions (e.g., Skillman \& Kennicutt 1993).

(b) Is the Predicted D and/or ${ }^{3} \mathrm{He}$ Yield Too Low?

A second possible crisis involves recent data from a QSO absorption system where - possibly - deuterium has been observed (Songaila et al. 1994, Carswell et al. 1994). The caveat is that there is no way to distinguish an individual absorption system due to D from one due to a hydrogen "interloper" (Steigman 1994b). If, however, the observed absorption features are, indeed, due to deuterium, the derived abundance is surprisingly high $\left(\mathrm{D} / \mathrm{H} \sim 2 \times 10^{-4}\right)$, nearly an order of magnitude higher than those derived from interstellar (Linsky et al. 1993) and/or solar system (Geiss 1993) data. Even so, this is not a crisis for BBN (Steigman 1994a,b) since such a high primordial $\mathrm{D}$ abundance forces us to a low value of $\eta$, reducing the (possibly problematic) predicted yield of ${ }^{4} \mathrm{He}$. Indeed, for $(\mathrm{D} / \mathrm{H})_{B B N} \approx 2 \times 10^{-4}$, we obtain $\eta_{10} \approx$ 1.5 and $Y_{B B N} \approx 0.23$ [as well as $\left({ }^{7} \mathrm{Li} / \mathrm{H}\right)_{B B N} \approx 2 \times 10^{-10}$, which is entirely consistent with the observational data]. This lower value of $\eta$ leads to a lower $\Omega_{B B N}$ which remains consistent with $(\geq) \Omega_{L U M}$, but which reinforces the evidence for non-baryonic dark matter $\left(\Omega_{B B N}<\Omega_{D Y N}\right)$. However, such a high primordial D abundance does 
challenge models of stellar and galactic chemical evolution since it would require that $\sim 90 \%$ of the pregalactic deuterium would have had to be destroyed in stars while avoiding the overproduction of ${ }^{3} \mathrm{He}$ (Steigman 1994b). Anticipated (and rumored!) additional data on possible D in other QSO absorption systems from Keck and the HST will help resolve - or sharpen - this potential crisis.

It is, however, the third crisis - the x-ray cluster baryon crisis - we wish to consider more fully here.

\section{THE X-RAY CLUSTER CRISIS}

Let's suppose for the moment that rich clusters of galaxies provide a "fair sample" of baryonic vs. non-baryonic mass in the Universe. Then the baryon fraction of the total mass in a cluster should be the same as the universal baryon fraction:

$$
f_{B} \equiv\left(M_{B} / M_{T O T}\right)_{\text {clusters }}=\Omega_{B} / \Omega
$$

Many rich clusters are x-ray sources, the emission being due to a hot intracluster gas (of baryons and electrons!). For such clusters (Loewenstein 1994) the x-ray emission provides information on the mass in hot gas (from the observed angular distribution of temperature and surface brightness) and on the total mass (from imposing a requirement of thermal hydrostatic equilibrium on the gas). In addition to the hot gas, baryons are, of course, to be found in the cluster galaxies. However, for most observed rich clusters the hot gas apparently dominates the baryon budget. In any case, ignoring the baryonic contribution by galaxies leads to a bound on $f_{B}\left(f_{H G} \leq f_{B}\right)$, so that

$$
\Omega \leq \Omega_{B} / f_{H G}
$$

Recent measurements show $f_{H G}$ to be rather large, $\sim 0.2$. The value of $f_{H G}$ inferred for an x-ray cluster depends on the distance to the cluster and, hence, on $H_{0}\left(f_{H G} \propto H_{0}^{-3 / 2}\right)$. If we define $f_{50}$ by

$$
f_{H G} \equiv f_{50} h_{50}^{-3 / 2}
$$

and use (2) for $\Omega_{B}$, we may write

$$
\Omega \leq 0.3 h_{50}^{-1 / 2}\left(\frac{0.20}{f_{50}}\right)\left(\frac{\eta_{10}}{4.0}\right) .
$$

From BBN we have concluded that $\eta_{10} \leq 4$, so that, for $H_{0} \geq 40, \Omega \leq(1 / 3)\left(0.2 / f_{50}\right)$ and, unless $f_{50}$ is $\ll 0.2$, we are led to conclude that $\Omega<1$, rejecting the popular Einstein-de Sitter cosmology. This, in a nutshell, is the x-ray cluster crisis.

White et al. (1993, "WNEF"), in an important paper, analyzed the data for the Coma cluster and derived $f_{50}$ (Coma) $\approx 0.14 \pm 0.04$. For $f_{50} \geq 0.10$ and $\eta_{10} \leq$ 4.0, $\Omega=1$ would require $H_{0} \leq 18 \mathrm{~km} \mathrm{~s}^{-1} \mathrm{Mpc}^{-1}$ ! Alternatively, for $H_{0} \geq 40$, we are led to infer $\Omega \leq 2 / 3$. 
How serious is this "crisis"? From $(7)$ it would appear that if $H_{0}$ and $f_{50}$ (Coma) are near the lower ends of their ranges $\left(H_{0} \approx 40, f_{50} \approx 0.1\right) \Omega=1$ could be recovered for $\eta_{10} \approx 6$. BBN theorists might be bullied into accepting this (Copi et al. 1994). However, the x-ray data suggest that the crisis is, in fact, much worse. In their analysis of Coma, WNEF were "conservative" in the sense of having chosen the largest of several estimates for the total mass. For example, had they used their mass estimate based on the assumption that the optical light traces the mass, the WNEF result would have been $f_{50}($ Coma $) \approx 0.23$.

There is, indeed, accumulating support for larger values of $f_{50}$. In their recent analysis of the Coma data, Fusco-Femiano \& Hughes (1994) find (within the Abell radius of $3 h_{50}^{-1} \mathrm{Mpc}$ ) $f_{50} \approx 0.27$. For the cluster Abell 478, White et al. (1994) find (within a radius of $2.3 h_{50}^{-1} \mathrm{Mpc}$ ) $f_{50} \geq 0.28$.

Even earlier, Fabian (1991), in his analysis of the core of the Shapley supercluster, concluded that $f_{50}>0.18$. Böhringer (1994) concludes from ROSAT data that three massive clusters (Coma, Perseus and A2256) are very similar to one another and have $0.12<f_{50}<0.45$. (The range here arises from uncertainty in the modelling, because ROSAT does not give good information on the temperature distributions.) Higher baryonic fractions receive further support from recent reanalyses of extant x-ray cluster data (Durret et al. 1994, White \& Fabian 1994). In both recent studies there is a clear trend of $f_{50}$ increasing with cluster size. The data of White \& Fabian (1994) for 19 clusters suggest that $f_{50} \approx 0.12 R_{50}^{0.6}$ where $R_{50}$ in Mpc, for each cluster, is the largest radius observed (for $H_{0}=50$ ). Evaluating this at the Abell radius of Coma $\left(R_{50}=3 \mathrm{Mpc}\right)$ gives $f_{50} \approx 0.23$. Finally, data from the ASCA satellite, which gives good information on the temperature distributions, imply that for three rich regular clusters (A496, A1795 and A2199), $f_{50} \approx 0.20$ within $\sim 0.75 h_{50}^{-1} \mathrm{Mpc}$, rising to $f_{50} \approx 0.23$ within $\sim 2 h_{50}^{-1} \mathrm{Mpc}$ (Mushotzky 1995). The baryons in the cluster galaxies, which we have neglected, would increase any of these $f_{50}$ values by 0.01-0.04 (WNEF, Mushotzky 1995).

Thus, it would appear that the x-ray cluster baryon crisis is worse, by a factor of $\sim 2$, than that identified for Coma by WNEF. For $f_{50} \geq 0.2$, we are driven to

$$
\Omega h_{50}^{1 / 2} \leq 0.3\left(\eta_{10} / 4\right) \text {. }
$$

From this perspective it seems far less likely that an overly restrictive BBN limit $\eta_{10} \leq 4$ is the "culprit" since, for $H_{0} \geq 40$ (80; Freedman et al. 1994), $\eta_{10} \geq 12(17)$ would be required to "save" $\Omega=1$. For such large values of $\eta$ the BBN yields of D and ${ }^{3} \mathrm{He}$ are far below their observed values while, far more seriously, the abundances of ${ }^{4} \mathrm{He}$ and ${ }^{7} \mathrm{Li}$ exceed those observed. 


\section{MITIGATING THE CRISIS}

Let's search for ways to mitigate this crisis. One or more of the following possibilities might contribute to a solution. We discuss several of these in more detail elsewhere (Felten \& Steigman 1994).

(a) Fair Sample?

The fair-sample hypothesis is an obvious point of attack. WNEF studied this by defining a "baryon enhancement factor" $\Upsilon$, replacing (4) with

$$
f_{B} \equiv\left(M_{B} / M_{T O T}\right)_{\text {cluster }}=\Upsilon \Omega_{B} / \Omega
$$

We can imagine that clustering processes could produce $\Upsilon>1$ or $<1$, in individual clusters or in all clusters. From (8), a value $\Upsilon \approx 3(4)$ for $H_{0}=50(80)$ would dispel the crisis. However, modern simulations by WNEF with $\Omega=1$ and cold dark matter (CDM) produce $\Upsilon \leq 1.4$, and in fact $\Upsilon$ tends to drop below unity at large cluster radii. Cen \& Ostriker (1993) found $\Upsilon \approx 2 / 3$ for Coma-like clusters in their simulations. This is a generic property and is likely to persist in other simulations with $\Omega<1$ and/or non-zero cosmological constant $\Lambda$. The reason is that gas can support itself partially against collapse through pressure, turbulence and shocks, but cold dark matter cannot. On smaller scales ( $\leq 100 \mathrm{kpc}$, the scales of galaxies), where the gas density can rise high enough for cooling to become important, the gas can indeed concentrate, but this does not happen on scales as large as $1 \mathrm{Mpc}$.

Larger values of $\Upsilon$ can be obtained by using cold plus hot dark matter (CHDM) simulations, still with $\Omega=1$ (Bryan et al. 1994, Primack et al. 1994). Hot dark matter can stay out of the clusters. The trouble is that HDM cannot dominate; otherwise the right kind of structure is not produced at the right times. The maximum $\Omega_{H D M}$ is about $0.2-0.3$. This is so small that even if all the HDM avoids the clusters, $\Upsilon$ cannot be large. People doing these simulations tend to press BBN theory rather hard. For example, Bryan et al. (1994) took $\eta_{10} \approx 7$, which may be unacceptable for BBN. Even with $\eta_{10}$ this large, Klypin (1995) reports that the maximum $f_{50}$ for clusters obtained in the simulations is about 0.15 . This is still too small to match the recent observations.

All the simulations above use Gaussian fluctuations as seeds to grow structure. Schramm (1994) and White (1995) have suggested that topological seeds (strings, etc.) in a HDM-dominated Universe $\left(\Omega_{H D M} \approx 0.7-0.8\right.$, perhaps $)$ might grow the right kind of structure and also produce large $\Upsilon$. This is a possibility, but we have not seen any such simulations.

(b) $H_{0}=30 ?$

Bartlett et al. (1994) suggest that a resolution is to be found in a very small Hubble constant; their favored value is $H_{0}=30$. From Figure 1 it is clear that $\Omega_{B B N}$ can reach the value of $f_{50} \approx 0.2$, doing away with the need for a baryon enhancement factor $\Upsilon$, if $H_{0}$ takes some small value. Observationally, we think that $H_{0}=30$ is a counsel of despair (cf. Freedman et al. 1994). Furthermore, $H_{0}=30$ is 
not small enough. From (8), for $H_{0}=30$, we obtain $\Omega=1$ only if $\eta_{10} \approx 10$, which is untenable.

(c) Magnetic Fields, Turbulence, Clumping

Lensing determinations of total masses of clusters give, in a few cases, masses higher by factors 2-2.5 than the total masses obtained in the x-ray analyses (MiraldaEscudé \& Babul 1994, Bartelmann \& Narayan 1995). If the x-ray total masses are too small by this factor, that would go more than halfway toward resolving the crisis. This has given rise to the suggestion that the x-ray assumption of thermal hydrostatic equilibrium is wrong. If magnetic pressure, for example, is in equipartition with thermal pressure in the hot gas, we can add this to the hydrostatic equation. The mass of hot gas remains the same, but the total (gravitational) mass inferred doubles, so that $f_{50}$ is reduced by a factor 2 . If turbulence is also in equipartition, the total mass triples.

The fields needed are large. Loeb \& Mao (1994), requiring equipartition, predict specifically $B \sim 50 \mu G$ in the cluster A2218, where there are no measurements of $B$. This may be important in a few clusters, but we doubt that it can provide a general solution to the crisis. The Coma cluster field is not nearly large enough (Kim et al. 1990), and cluster fields generally are thought to be $\sim 1-2 \mu G$ (Kronberg 1994).

The lensing determinations have their own problems, and need refinements in some cases (Bartelmann \& Narayan 1995). Cluster masses have also been determined by the familiar use of galaxy velocity dispersions ("virial-theorem masses"). These are subject to factor-of-2 systematic errors arising from models of orbit shapes and of the radial mass distributions, but (within errors) they agree with the $\mathrm{x}$ ray masses (WNEF, Mushotzky 1995). The galaxies (unlike the gas) cannot be supported by $B$-fields and turbulence, so the agreement between x-ray and virialtheorem masses puts some limit on the importance of fields and turbulence.

There is also a related suggestion that the gas may be strongly clumped. Since the emissivity of the gas goes as $n^{2}$, clumping increases the pressure but reduces the mass of gas required (WNEF) and reduces $f_{50}$. One might then expect to see a superposition of many different temperatures (in different clouds) in the $\mathrm{x}$ - ray data. There is no evidence of this. We also have to question the stability of such a clumped system. If some kind of equilibrium is maintained, then something, e.g., a large $B$-field (> $>0 \mu G$ in A2218!), must supply pressure in the voids yet remain outside the clouds - astrophysically a bizarre situation. If there is no equilibrium, we could think of the clouds as moving freely on orbits. In either case, the clouds might dissipate rapidly. And in either case, the calculation of total mass from $\mathrm{x}-$ ray data would be erroneous, and the existing agreement between the x-ray and virial-theorem masses would be an accident (WNEF). 


\section{COSMOLOGY AND DICKE COINCIDENCES}

We come now to the possibility that the "standard", i.e., Einstein-de Sitter, cosmology should be abandoned. To comment on this, we write the Friedmann equation:

$$
1=\frac{8 \pi G \rho_{M 0}}{3 H^{2}}\left(\frac{a_{0}}{a}\right)^{3}-\frac{c^{2} K_{0}}{H^{2}}\left(\frac{a_{0}}{a}\right)^{2}+\frac{\Lambda}{3 H^{2}},
$$

where $\rho_{M}$ is mass density, $a(t)$ is the scale factor, $K$ is the Gaussian curvature of three-space, subscripts 0 denote present values, and $H(t)$ is the Hubble function $(\dot{a} / a)$. (The dependence of the first term above on $a$ steepens to the Lemaitre form $\left(a_{0} / a\right)^{4}$ in the early radiation-dominated universe.) The three terms have familiar shorthand forms (Peebles 1993, p. 100):

$$
1=\Omega_{M}+\Omega_{K}+\Omega_{\Lambda} .
$$

( $\Omega_{M 0}$ is the quantity we have called $\Omega$ earlier. We switch notation to discuss the three terms more easily.)

Many cosmologists are attached to the Einstein-de Sitter model, in which the last two terms are negligible at present. One still hears often (e.g., Fujii \& Nishioka 1991) the claim that these two terms must be negligible to avoid the curse of the "Dicke coincidences" (Dicke \& Peebles 1979). If Einstein's gravity is correct, we know astronomically that at present $\Omega_{M 0}$ is not terribly small $\left(\ll 10^{-2}\right)$ and the other two terms are not terribly large $(>>>1)$. Because these two terms shrink relative to $\Omega_{M}$ as we look back into the past $(a \rightarrow 0)$, it follows that $\Omega_{M}$ was extremely close to unity at early epochs (e.g., at epoch "b", the epoch of BBN). This is the "flatness problem". Many believe that the only way to explain this fantastic coincidence is to postulate that $\Omega_{M b}$ was (and $\Omega_{M 0}$ remains) exactly unity. This would agree with the idea that $\Omega_{K}$ was driven to zero by inflation, and that $\Omega_{\Lambda}$ is zero because of some unknown physics.

But in fact, $\Omega_{M b}$ must be close to unity in any cognizable ("anthropic") Universe (Barrow \& Tipler 1986, pp. 408 ff.; Peebles 1993, pp. 364 ff.). Consider, for a moment, only the familiar $\Lambda=0$ models. If $\Omega_{M b}$ is appreciably below unity, the early Universe goes into a linear expansion in a few doubling times (i.e., a few minutes) and according to present ideas of cosmogony it could never form structure. If $\Omega_{M b}$ exceeds unity, the Universe recollapses, again in a few minutes. All of these models are hostile to life. Thus the anthropic principle gives a solution to the flatness problem without the postulate that $\Omega_{K b}$ is exactly zero.

Sizable $\Omega_{\Lambda b}$, it seems, would not add anthropic possibilities. Such a model could coast at a hot dense epoch, but upon emerging, it would go into fast recollapse or accelerated expansion. [The "flow" of the $\Omega$ terms has been studied (e.g., Madsen \& Ellis 1988, Ehlers \& Rindler 1989, Cho \& Kantowski 1994).]

Anthropic arguments like these discomfit some physicists, probably be cause advocates sometimes bring in teleological and even theological baggage. Kolb \& 
Turner (1990), writing in their entertaining style, call the anthropic principle "lame" (p. 269), but then relent and concede (p. 315) that it has "some rational basis". We believe that anthropic arguments can be quite powerful and are certainly worthy to answer "coincidence" arguments.

Consider now the present (epoch "0") values of the terms in (11). We grant, because of the argument above, that $\Omega_{K 0}$ and $\Omega_{\Lambda 0}$ need not be exactly zero, but we note that all three terms have different $a$-dependences. Is it then a big coincidence if more than one of the terms are $\sim 1$ at present? Consider $\Omega_{\Lambda 0}$. The physically "natural" value for $\Omega_{\Lambda 0}$ (Weinberg 1989; Carroll, Press, \& Turner 1992) seems to be $\sim \pm 10^{118}$ ! We could imagine that there is a natural distribution of possible magnitudes with this as mean. But anthropically $\Omega_{\Lambda 0}$ is limited roughly to

$$
-10<\Omega_{\Lambda 0}<100 \Omega_{M 0}
$$

(Weinberg 1987, 1989; cf. Barrow \& Tipler 1986, inequality 6.131). The argument is essentially the same as that given above. $\Omega_{\Lambda 0}<-10$ makes the Universe recollapse too quickly; $\Omega_{\Lambda 0}>100 \Omega_{M 0}$ prevents the growth of condensations (structure). The upper limit has been derived rigorously only for flat models, and there are some errors in published derivations. The numbers may change somewhat, but rough numbers are adequate to make our point, which is this: Once we realize that $\Omega_{\Lambda 0}$ is not free to roam up to $10^{118}$, but is constrained below 100 or so by the condition that intelligent life exists, the "coincidence" involved if $\Omega_{\Lambda 0} \sim 1$ is no longer so impressive. Weinberg (1989) suggests that the true value should be near the upper limit in (12) "because there is no anthropic reason for it to be any smaller". We wonder whether the "natural" probability distribution of $\Omega_{\Lambda 0}$ is established well enough to justify that statement. We think that an astronomically admissible and cosmogonically significant value (say, $0.2-0.7$ ) may be plausible.

This is already acknowledged, for many simulations have been done with $\Omega_{\Lambda 0}>0$. Should we also consider non-zero $\Omega_{K 0}$ ? Inflation requires $\Omega_{K 0}=0$; models in which inflation stops short of flattening the Universe encounter difficulties (Kashlinsky, Tkachev \& Frieman 1994). Inflation gives a nice solution to the horizon problem, but its predictive power has not been great. What if we abandon the inflationary model? Should we still maintain that $\Omega_{K 0}=0$ ? If all three terms in $(11)$ are $\sim 1$, is that an unacceptable "double coincidence"?

This is a bit murky, but several points should be made:

(1) $\Omega_{K 0}$ is not really free, being determined by (11).

(2) Nevertheless there are two free variables in (11), because $\Omega_{M 0}$ is free. In our Universe we know its value very roughly, but in other cognizable Universes its value might be very different. Note that it affects the upper limit in (12).

(3) In principle there are anthropic limits on the ratios of $\Omega_{K 0}$ to the other terms (Carter 1974, Carr \& Rees 1979), analogous to (12). $\Omega_{K 0}$ is not free to roam to extreme values. 
We have not seen a derivation of joint anthropic constraints on the terms in (11) when all three are allowed to be non-zero, but we can guess at the results. We believe that the case $\Omega_{K 0}=0$ deserves some preference because of inflation theory. In alternative models with $\Omega_{K 0} \neq 0$, we would guess that the case $\Omega_{\Lambda 0}=0$ is more likely than the case of all three terms $\sim 1$, but not by a large factor when anthropic constraints are considered. We cannot conclude safely from coincidence arguments that any of the terms in (11) is negligible. Anthropic constraints are numerous and powerful (Carter 1974, Carr \& Rees 1979, Barrow \& Tipler 1986); we have mentioned only the simplest ones.

Perhaps some of these ideas for mitigating the x-ray cluster crisis will be productive. Perhaps simulations with topological seeds and hot dark matter can produce the right kind of structure and achieve a baryon enhancement factor $\Upsilon \approx 3-4$, so that $\Omega_{M 0}=1$ can be maintained. If not, open-universe models will necessarily gain in popularity (Cen \& Ostriker 1993).

\section{ACKNOWLEDGEMENTS}

We wish to thank Sid Bludman, Richard Ellis, Andy Fabian, Sasha Kashlinsky, Rich Mushotzky, Martin Rees and Simon White for advice and discussion. J. E. F. is grateful to NASA for partial support under RTOP No. 188-44-53-05, and wishes

to thank the Aspen Center for Physics and all the participants in the Aspen 1994 Workshops on Gravitational Clustering and on Clusters of Galaxies. The work of G. S. at OSU is supported by DOE grant DE-FG02-94ER-40832. Some of this work was done when G. S. was an Overseas Fellow at Churchill College, Cambridge and a Visiting Fellow at the Institute of Astronomy and he thanks them for hospitality. G. S. thanks the organizers of the Gamow Seminar for their kind hospitality and Roger Chevalier for assistance in preparing this manuscript for the Proceedings. 


\section{REFERENCES}

Bartelmann, M., \& Narayan, R.: 1995, in "Dark Matter" (Proc. 5th Annual Astrophysics Conference in Maryland, Oct. 10-12, 1994), ed. S. S. Holt \& C. L. Bennett (New York: American Institute of Physics), in press

Barrow, J. D., \& Tipler, F. J.: 1986, "The Anthropic Cosmological Principle" (Oxford: Oxford University Press)

Bartlett, J. G., Blanchard, A., Silk, J., \& Turner, M. S.: 1994, Science, submitted

Boesgaard, A.M., \& Steigman, G.: 1985, ARA\&A, 23, 319

Böhringer, H.: 1994, preprint

Bryan, G. L., Klypin, A., Loken, C., Norman, M. L., \& Burns, J. O.: 1994, APJLett, 437, L5

Carr, B. J., \& Rees, M. J.: 1979, Nature, 278, 605

Carroll, S. M., Press, W. H., \& Turner, E. L.: 1992, ARA\&A, 30, 499

Carswell, R. F, Rauch, M., Weymann, R. J., Cooke, A. J., \& Webb, J. K.: 1994, $M N R A S, 268, \mathrm{~L} 1$

Carter, B.: 1974, in "Confrontation of Cosmological Theories with Observational Data" (IAU Symposium 63, Cracow, September 10-12, 1973), ed. M. S. Longair (Dordrecht: Reidel), 291

Cen, R., \& Ostriker, J. P.: 1993, ApJ, 417, 404

Cho, H. T., \& Kantowski, R.: 1994, Phys. Rev., D50, 6144

Copi, C. J., Schramm, D. N., \& Turner, M. S.: 1994, Science, submitted

Dearborn, D. S. P., Schramm, D. N., \& Steigman, G.: 1986, Ap. J. 302, 35

Dicke, R. H., \& Peebles, P. J. E.: 1979, in "General Relativity: An Einstein Centenary Survey", ed. S. W. Hawking \& W. Israel (Cambridge: Cambridge University Press), 504

Durret, F., Gerbal, D., Lachièze-Rey, M., Lima-Neto, G., \& Sadat, R.: 1994, A\&A, 287, 733

Efstathiou, G., Ellis, R. S., \& Peterson, B. A.: 1988, MNRAS, 232, 431

Ehlers, J., \& Rindler, W.: 1989, MNRAS, 238, 503

Fabian, A. C.: 1991, MNRAS, 253, 29P

Felten, J. E. \& Steigman, G.: 1994, in preparation

Freedman, W. L., et al.: 1994, Nature, 371, 757

Fujii, Y., \& Nishioka, T.: 1991, Phys. Lett., B254, 347 
Fusco-Femiano, R., \& Hughes, J. P.: 1994, ApJ, 429, 545

Geiss, J.: 1993, in "Origin and Evolution of the Elements", ed. N. Prantzos, E. Vangioni-Flam \& M. Cassé, (Cambridge: Cambridge University Press), 89

Kashlinsky, A., Tkachev, I.I., \& Frieman, J.: 1994, Phys. Rev. Lett., 73, 1582

Kim, K.-T., Kronberg, P. P., Dewdney, P. E., \& Landecker, T. L.: 1990, ApJ, 355, 29

Klypin, A.: 1995, in "Dark Matter" (Proc. 5th Annual Astrophysics Conference in Maryland, Oct. 10-12, 1994), ed. S. S. Holt \& C. L. Bennett (New York: American Institute of Physics), in press

Kolb, E. W., \& Turner, M. S.: 1990, "The Early Universe" (Redwood City, CA: Addison-Wesley)

Kronberg, P. P.: 1994, Rep. Prog. Phys., 57, 325

Linsky, J. L., et al.: 1993, ApJ, 402, 694

Loeb, A., \& Mao, S.: 1994, ApJ, 435, L109

Loewenstein, M.: 1994, ApJ, 431, 91

Madsen, M. S., \& Ellis, G. F. R.: 1988, MNRAS, 234, 67

Mather, J. C., et al.: 1994, ApJ, 420, 439

Miralda-Escudé, J., \& Babul, A.: 1994, ApJ, submitted

Mushotzky, R. F.: 1995, in "Dark Matter" (Proc. 5th Annual Astrophysics Confer ence in Maryland, Oct. 10-12, 1994), ed. S. S. Holt \& C. L. Bennett (New York: American Institute of Physics), in press

Olive, K. A., \& Steigman, G.: 1994, ApJS, in press

Pagel, B. E. J.: 1993, Proc. Nat. Acad. Sci., 90, 4789

Pagel, B. E. J., Simonson, E. A., Terlevich, R. J., \& Edmunds, M. G.: 1992, MNRAS, 255, 325

Peebles, P. J. E.: 1993, "Principles of Physical Cosmology" (Princeton: Princeton University Press)

Primack, J. R., Holtzman, J., Klypin, A., \& Caldwell, D. O.: 1994, Phys. Rev. Lett., submitted

Schramm, D. N.: 1994, private communication

Skillman, E. D., \& Kennicutt, R. C., Jr.: 1993, ApJ, 411, 655 
Skillman, E. D., Terlevich, R. J., Terlevich, E., Kennicutt, R. C., Jr., \& Garnett, D. R.: 1993, in "Texas/PASCOS '92: Relativistic Astrophysics and Particle Cosmology", ed. C. W. Akerlof \& M. A. Srednicki (Ann. N. Y. Acad. Sci., 688) (New York: New York Academy of Sciences), 739

Songaila, A., Cowie, L. L., Hogan, C. J., \& Rugers, M.: 1994, Nature , 368, 599

Steigman, G.: 1994a, preprint OSU-TA-22/94. To appear in the proceedings of the ESO/EIPC Workshop on "The Light Element Abundances", ed. P. Crane (Berlin: Springer) $\therefore$ 1994b, MNRAS, 269, L53

Steigman, G., \& Tosi, M.: 1992, ApJ 401, 150 $\therefore$ 1994, ApJ, submitted

Walker, T. P., Steigman, G., Schramm, D. N., Olive, K. A., \& Kang, H.-S.: 1991, ApJ, 376, 51 (WSSOK)

Weinberg, S.: 1987, Phys. Rev. Lett., 59, 2607

_. 1989, Rev. Mod Phys., 61, 1

White, D. A., \& Fabian, A. C.: 1994, MNRAS, in press

White, D. A., Fabian, A. C., Allen, S. W., Edge, A. C., Crawford, C. S., Johnstone, R. M., Stewart, G. C., \& Voges, W.: 1994, MNRAS, 269, 589

White, S. D. M.: 1995, in "Dark Matter" (Proc. 5th Annual Astrophysics Conference in Maryland, Oct. 10-12, 1994), ed. S. S. Holt \& C. L. Bennett (New York: American Institute of Physics), in press

White, S. D. M., Navarro, J. F., Evrard, A. E., \& Frenk, C. S.: 1993, Nature 366, $429(\mathrm{WNEF})$

Yang, J., Turner, M. S., Steigman, G., Schramm, D. N., \& Olive, K. A.: 1984, ApJ, $\mathbf{2 8 1}, 493$ 


\section{FIGURE CAPTION}

FIG. 1 - The range of total baryonic mass density $\Omega_{B B N}$ allowed by BBN theory and element abundances (for $2.8 \leq \eta_{10} \leq 4.0$ ), shown as a function of assumed Hubble constant $H_{0}\left(\mathrm{~km} \mathrm{~s}^{-1} \mathrm{Mpc}^{-1}\right)$. We also show a generous estimate for the mass density $\Omega_{L U M}$ due to baryons in the luminous parts of galaxies, and a lower limit to the total gravitating mass density $\Omega_{D Y N}$ implied by large-scale dynamical studies. 
This figure "fig1-1.png" is available in "png" format from: http://arxiv.org/ps/astro-ph/9502029v1 


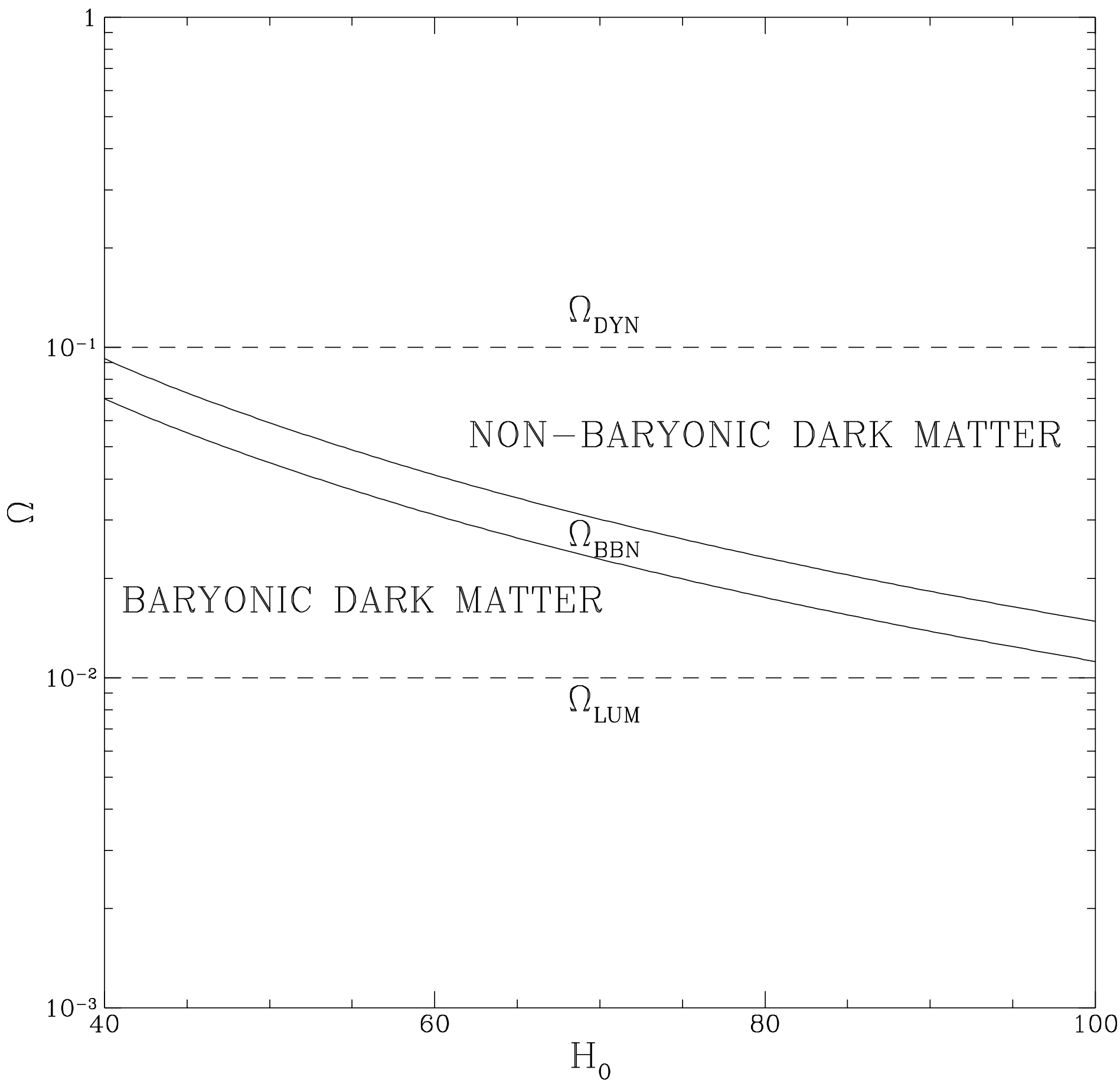

